\title{
ICHTHYOFAUNAL DIVERSITY OF THE FRESHWATER SWAMP FOREST RATARGUL, SYLHET, BANGLADESH
}

\author{
Shamima Nasren ${ }^{1 *}$, Shuvashis Talukder ${ }^{1}$, Partho Protim Barman ${ }^{2}$ and Md. Abdullah Al Mamun \\ ${ }^{1}$ Department of Fish Biology and Genetics, Sylhet Agricultural University, Sylhet-3100, Bangladesh \\ ${ }^{2}$ Departmnet of Coastal and Marine Fisheries, Sylhet Agricultural University, Sylhet-3100, Bangladesh \\ ${ }^{3}$ Department of Fish Health Management, Sylhet Agricultural University, Sylhet-3100, Bangladesh \\ *e-mail: s.nasren2013@gmail.com, Tel: +8801718903436/Fax: +88 0821761980
}

\begin{abstract}
An ichthyofaunal study in the Ratargul swamp forest, north Sylhet was carried out from July 2014 to June 2015 resulted in the collection of 37 species of fishes belonging to nine orders and 21 families. Order Cypriniformes dominated contributing $27.03 \%$ of the total fish. Shannon-Wiener diversity index $\left(H^{\prime}\right)$, the Margalef species richness $(d)$, the Pielou's evenness index $\left(J^{\prime}\right)$ and the Simpson dominance index $(c)$ were calculated as $2.28 \pm 0.294,5.18 \pm 1.23,0.96 \pm 0.013$ and $0.96 \pm 0.012$. SIMPER analysis divulged the highest dissimilarity $(56.22 \%)$ in between the January-August group and lowest $(10.07 \%)$ in the July-August group. Non metric-multidimensional scaling (nMDS) showed 50\% similarity for all months, while 80\% similarity showed four separate clusters among all months. Wise use of this wetland and establishment of fish sanctuary are suggested for long-term conservation of the native fish species and the ecosystem.
\end{abstract}

KEYWORDS: Ratargul swamp forest, Ichthyofaunal diversity, Cypriniformes, Diversity indices, SIMPER analysis, nMDS and Conservation

\section{INTRODUCTION}

In terms of aquatic biodiversity, Bangladesh possesses the third largest position (about 800 species in fresh, brackish and marine waters) in Asia ${ }^{1}$. The geographic location of Bangladesh is helpful and it gives huge opportunities to keep up prospects in fisheries sector ${ }^{2}$. Bangladesh has many flood plains in the form of saucershaped wetlands such as haors, baors and marshy or swampy water areas in the northeastern region of the country, like in Sylhet division (Habiganj, Moulvibazar, Sunamganj and Sylhet districts). Ratargul swamp forest is the only forested fresh water wetland of Bangladesh and it is among twenty-two (22) freshwater swamp forests of the world. Nabahungu ${ }^{3}$ mentioned freshwater swamp forest as 'biological supermarket' for its unique biodiversity. This ecosystem harbors rich species diversity with a number of timber and non- timber forest products which acts an important factor in maintaining biodiversity and same time sustaining livelihoods of local people ${ }^{4}$. Fish hold a large part of standing biomass of aquatic ecosystem (Fresh, Brackish and Marine water ecosystem) ${ }^{5}$. A large number of fresh water fish species already have entered into endangered list, for intense human intervention like, habitat degradation and $\operatorname{loss}^{6,7}$ and freshwater ecosystem of Bangladesh is not out of these scenarios ${ }^{8-11}$. Research are being carried out worldwide to build conservation planning to preserve freshwater fishes ${ }^{6,12-15}$. Although, 
Ichthyofaunal diversity of Ratargul has been made by Islam $^{16}$ but these studies are inadequate to discuss the crucial issues related to conserve fishes in the swamp forest. In this context, the present study was carried out (a) to find out the current pattern of ichthyofaunal diversity and abundance, and (b) to construct recommendations for ichthyofaunal conservation.

\section{MATERIALS AND METHODS}

\section{Sampling}

Ratargul swamp forest (latitude $25^{\circ} 00.025^{\prime} \mathrm{N}$ and longitude $91^{\circ} 58.180^{\prime} \mathrm{E}$ ) is situated on the bank of the Goyain river and located at about 45 kilometers from Sylhet city (Figure 1). The Ratargul swamp forest covers an area of 30,325 acres area where 504 acres declared as the sanctuary in 1973. The word, "Rata" is one kind of tree, locally known the forest's name comes from used by the locals of Sylhet. This swamp forest is surrounded by nine villages of Fateh Pur union namely Ratarghul, Bagh Bari, Dewaner Gaon, Ram Nagar, Mashkher, Julurpar, Chailtha Bari, Kucharkandi and Erertuk under. Primary data on current pattern of ichthyofaunal diversity and abundance collected twice in a month for one year period. Ichthyofaunal diversity was studied and observed through the collection unsorted fish sample directly from the fishermen. The following formulas were used to calculate the relative fish abundance in this research.

Number of samples of particular species $x$ 100/ Total number of samples
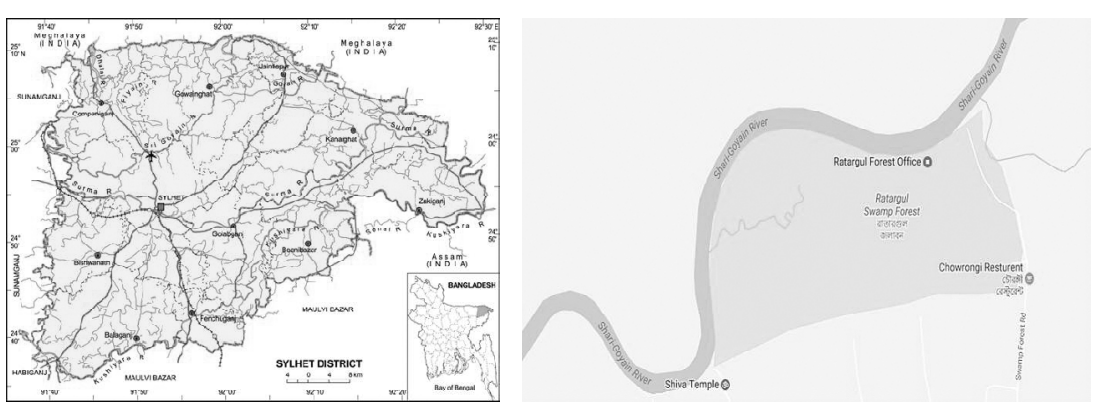

Figure 1: Map of the Ratargul swamp forest area showing the location of the study area

The collected fish samples were immediately preserved in ice box and transferred to the Laboratory of Fish Biology and Genetics Department, Sylhet Agricultural University, Sylhet. Collected sample were sorted, rinsed with tap water and identified based on morphological characteristics $^{17-21}$.

\section{Analysis}

Shannon-Wiener index $(H 2)^{22}$ was used to analyze the fish diversity in the species assemblage, Margalef index $(d)^{23}$ was used to measure the species richness, 
Pielou's index $(J 2)^{24}$ was used to determine evenness and dominance by Simpson dominance index $(c)^{25}$.

Shannon-Wiener diversity index $\left(H^{\prime}\right)$ :

$H^{\prime}=$ - Sum [pi x log (pi)]

Where,

$H^{\prime}=$ Shannon-Wiener index

$\mathrm{pi}=\mathrm{n}_{\mathrm{i}} / \mathrm{N}$

$\mathrm{n}_{\mathrm{i}}=$ no. of individuals of a species

$\mathrm{N}=$ Total number of individuals

\section{Pielou's evenness index $(J ')$ :}

Where,

$\mathrm{H}(\mathrm{s})=$ the Shannon-Wiener information function.

$\mathrm{H}(\max )=$. the theoretical maximum value for $\mathrm{H}(\mathrm{s})$ if all species in the sample were equally abundant.

\section{Simpson dominance index $(c)$ :}

Where, $\mathrm{n}_{\mathrm{i}}=$ number of individuals in the 'each' species

$\mathrm{N}=$ total number of individuals

$\mathrm{S}=$ total number of species

All calculation were done using the PRIMER V6 (version 6.1) software.

The significance of temporal variation was found by One-way analysis of Similarity (ANOSIM) in the structure of fish assemblage $^{26,27}$. A Bray-Curtis similarity matrix test was used where $\log (\mathrm{X}+1)$ transformed data were calculated. Similarity percentages analyses (SIMPER) Clarke ${ }^{26}$ were used for finding the average nonsimilarity in the contribution of every taxon. Hierarchical agglomerative clustering with group-averaging linking and nMDS or nonmetric multidimensional scaling was performed to investigate months similarities among fish abundance and analysis were done on the Bray-Curtis similarity ${ }^{28}$. For descriptive statistics and ANOVA, test SPSS (Statistical Package for Social Sciences) software V2029 were used. The software PRIMER V6 version 6.1(Plymouth Routines in Multivariate Ecological Research) ${ }^{26,27}$ was used for multivariate analyses.

\section{RESULTS AND DISCUSSION}

\section{Fish species of Ratargul swamp forest}

A sum of 37 fish species from nine orders and 21 families were reported from the study area (Table 1). Order Cypriniformes dominated contributing $27.03 \%$ of the fish assemblage, next were Siluriformes $(24.32 \%)$, Perciformes (16.22\%), Anabantiformes (10.81\%), Clupeiformes $(8.11 \%)$, Synbranchiformes (5.41\%), Gobiiformes (2.70\%), Mugiliformes $\quad(2.70 \%) \quad$ and Tetraodontiformes (2.70\%) (Figure 2).

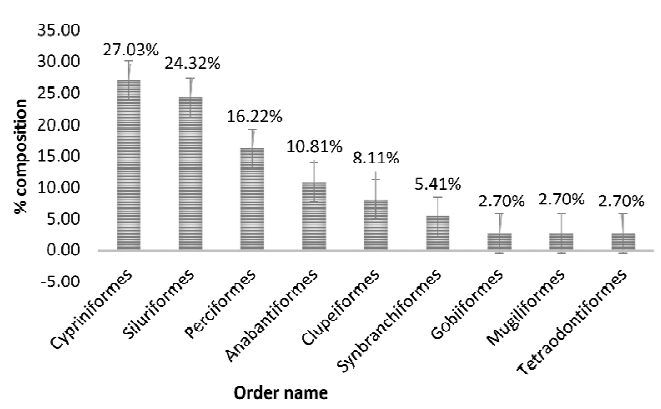

Figure 2: Order-wise composition of fishes in the study area 
Table 1. List of different fish species with their order, family, scientific name, common name, local name, and IUCN status.

\begin{tabular}{|c|c|c|c|c|c|c|}
\hline \begin{tabular}{|l|} 
Sl. \\
No.
\end{tabular} & Order & Family & Scientific Name & English Name & Local Name & $\begin{array}{l}\text { IUCN } \\
\text { Status* }\end{array}$ \\
\hline \multirow{4}{*}{$\begin{array}{l}1 \\
2 \\
3 \\
4\end{array}$} & \multirow[t]{4}{*}{ Clupeiformes } & \multirow[t]{3}{*}{ Clupeidae } & Gudusia chapra (Hamilton, 1822) & Indian river shad & Chapila & NT \\
\hline & & & Tenualosa ilisha (Hamilton, 1822) & Indian river shad & Ilish & NT \\
\hline & & & Corica soborna (Hamilton, 1822) & Ganges river sprat & Kachki & NT \\
\hline & & Cobitidae & Lepidocephalus guntea (Hamilton, 1822) & Guntea loach & Gutum & NT \\
\hline 5 & \multirow[t]{9}{*}{ Cypriniformes } & \multirow[t]{9}{*}{ Cyprinidae } & $\begin{array}{l}\text { Salmostomata acinaces } \\
\text { (Valenciennes,1844) }\end{array}$ & $\begin{array}{l}\text { Silver razor } \\
\text { belly minnow }\end{array}$ & Chella & NT \\
\hline 6 & & & Labeo calbasu (Hamilton, 1822) & Black rohu & Kalibaush & EN \\
\hline 7 & & & Labeo gonius (Hamilton, 1822) & Kuria labeo & Ghonia & EN \\
\hline 8 & & & Labeo bata (Hamilton, 1822) & Bata & Bata & NT \\
\hline 9 & & & Osteobrama cotio (Hamilton, 1822) & Cotio & Dhela & EN \\
\hline 10 & & & $\begin{array}{l}\text { Amblypharyngodon mola } \\
\text { (Hamilton, 1822) }\end{array}$ & Mola carplet & Mola & NT \\
\hline 11 & & & Puntius ticto (Hamilton, 1822) & Firefin barb/ticto barb & Tit punti & VU \\
\hline 12 & & & Puntius sophore (Hamilton, 1822) & Spot fin swamp barb & Jat punti & NT \\
\hline 13 & & & Esomus danrica (Hamilton, 1822) & $\begin{array}{l}\text { Gangetic scissortail } \\
\text { rasbora }\end{array}$ & Darkina & DD \\
\hline 14 & Gobiiformes & Oxudercidae & Awaous grammepomus (Bleeker,1849) & Scribbled Goby & Goby & VU \\
\hline \multirow{4}{*}{$\begin{array}{l}15 \\
16 \\
17 \\
18\end{array}$} & \multirow[t]{4}{*}{ Anabantiformes } & Anabantidae & Anabas testudineus (Bloch, 1792) & Climbing perch & Koi & NT \\
\hline & & \multirow[t]{3}{*}{ Channidae } & Channa striatus (Bloch, 1793) & Snakehead murrel & Shol & NT \\
\hline & & & Channa punctatus (Bloch, 1793) & Spotted snakehead & Taki & NT \\
\hline & & & $\begin{array}{l}\text { Channa orientalis } \\
\text { (Bloch \& Schneider, 1801) }\end{array}$ & Asiatic snakehead & Cheng & VU \\
\hline 19 & \multirow[t]{6}{*}{ Perciformes } & \multirow[t]{2}{*}{ Centropomidae } & Chanda nama (Hamilton, 1822) & High finglassy perchlet & Lomba chanda & VU \\
\hline 20 & & & Parambassis ranga (Hamilton, 1822) & High finglassy perchlet & Lal chanda & VU \\
\hline 21 & & Sciaenidae & Otolithoides pama (Hamilton, 1822) & Pama croaker & Pama & NT \\
\hline 22 & & Nandidae & Nandus nandus (Hamilton, 1822) & Gangetic leaf fish & Meni/veda & VU \\
\hline 23 & & Osphronemidae & Ctenops nobilis (McClelland, 1845) & Frail Gourami & Neftani & EN \\
\hline 24 & & Sciaenidae & Johnius coitor(Hamilton, 1822) & Coitor croaker & Poa & NT \\
\hline 25 & Mugiliformes & Mugilidae & Rhinomugil corsula(Hamilton, 1822) & Corsula mullet & Halla & NT \\
\hline 26 & \multirow[t]{4}{*}{ Siluriformes } & Plotosidae & Plotosus canius(Hamilton, 1822) & Gray eel catfish & Gang magur & VU \\
\hline 27 & & \multirow[t]{3}{*}{ Bagridae } & Mystus bleekeri (Day,1877) & Gantatic mystus & Gulsha & NT \\
\hline 28 & & & Mystus tengra(Hamilton, 1822) & Stripped dwarf catfish & Bujuri tengra & NT \\
\hline 29 & & & Mystus vittatus (Bloch,1794) & Striped dwarf catfish & Tengra & NT \\
\hline
\end{tabular}




\begin{tabular}{|l|l|l|l|l|l|l|}
\hline $\begin{array}{l}30 \\
31\end{array}$ & & Sisoridae & Bagarius bagarius(Hamilton, 1822) & Gangetic goonch & Baghair & CR \\
\cline { 3 - 7 } 32 & & Heteropneustidae & Heteropneustes fossilis (Bloch,1794) & Stinging catfish & Shing & NT \\
\cline { 3 - 7 } 33 & Schilbeidae & Eutropiichthys vacha(Hamilton, 1822) & Batchwa bacha & Bacha & CR \\
\cline { 3 - 7 } 34 & Ailiidae & Ailia coila (Hamilton, 1822) & Gangetic ailia & Kajuli & NT \\
\cline { 3 - 7 } 35 & Synbranchiformes & Mastacembelidae & Macrognathus aculeatus(Bloch,1786) & One striped spiny eel & Tara baim & VU \\
\cline { 3 - 7 } 36 & & & Mastacembelus armatus (Lacepede,1800) & Tire-track spiny eel & Shal baim & EN \\
\hline 37 & Tetraodontiformes & Tetraodontidae & Dichotomyctere fluviatalis (Hamilton, 1822) & Green pufferfish & Potka & NT \\
\hline
\end{tabular}

*Status DD,CR, EN, NT and VU are based on IUCN Bangladesh (2015); DD, Data deficient; CR,Critically Endangered; EN, Endangered; NT, Not Threatened and VU, Vulnerable.

Table 2. SIMPER analysis showing \% dissimilarity between different months with their contributing fish species

\begin{tabular}{|c|c|c|c|c|c|}
\hline \multicolumn{2}{|c|}{$\begin{array}{l}\text { January \& February } \\
\text { Average dissimilarity }=17.76 \%\end{array}$} & \multicolumn{2}{|c|}{$\begin{array}{l}\text { Groups January \& March } \\
\text { Average dissimilarity }=\mathbf{2 6 . 7 6}\end{array}$} & \multicolumn{2}{|c|}{$\begin{array}{l}\text { Groups February \& March } \\
\text { Average dissimilarity = 20.89 }\end{array}$} \\
\hline Species & Cont. $(\%)$ & Species & Cont. $(\%)$ & Species & Cont. $(\%)$ \\
\hline M. aculeatus & 18.90 & M. tengra & 14.66 & M. tengra & 16.04 \\
\hline H. fossilis & 12.79 & C. nama & 10.44 & C. nama & 11.43 \\
\hline N. nandus & 12.79 & H. fossilis & 10.44 & N. nandus & 9.06 \\
\hline A. testudinious & 12.79 & C. soborna & 8.28 & C. srtiatus & 9.06 \\
\hline G. chapra & 8.07 & C. srtiatus & 8.28 & A. testudinious & 9.06 \\
\hline A. mola & 8.07 & M. aculeatus & 7.12 & C. soborna & 8.49 \\
\hline M.vittatus & 6.51 & M. vittatus & 6.90 & M. armatus & 6.99 \\
\hline M. bleekeri & 5.47 & A. mola & 6.90 & G .chapra & 5.72 \\
\hline C. nama & 3.92 & M. bleekeri & 5.94 & C. orientalis & 5.72 \\
\hline C. punctatus & 3.35 & M. armatus & 5.22 & M. aculeatus & 5.6 \\
\hline \multicolumn{2}{|c|}{$\begin{array}{l}\text { January \& April } \\
\text { Average dissimilarity }=35.65\end{array}$} & \multicolumn{2}{|c|}{$\begin{array}{l}\text { February \& April } \\
\text { Average dissimilarity }=33.95\end{array}$} & \multicolumn{2}{|c|}{$\begin{array}{l}\text { Groups March \& April } \\
\text { Average dissimilarity }=20.11 \\
\end{array}$} \\
\hline \multicolumn{2}{|c|}{$\begin{array}{l}\text { Groups Jan \& May } \\
\text { Average dissimilarity }=40.23\end{array}$} & \multicolumn{2}{|c|}{$\begin{array}{l}\text { Groups January \& November } \\
\text { Average dissimilarity }=37.30\end{array}$} & \multicolumn{2}{|c|}{$\begin{array}{l}\text { Groups February \& November } \\
\text { Average dissimilarity }=38.85\end{array}$} \\
\hline \multicolumn{2}{|c|}{$\begin{array}{l}\text { Groups Mar \& May } \\
\text { Average dissimilarity }=29.30\end{array}$} & \multicolumn{2}{|c|}{$\begin{array}{l}\text { Groups April\& May } \\
\text { Average dissimilarity=14.37 }\end{array}$} & \multicolumn{2}{|c|}{$\begin{array}{l}\text { Groups January\& June } \\
\text { Average dissimilarity }=43.15\end{array}$} \\
\hline \multicolumn{2}{|c|}{$\begin{array}{l}\text { Groups February \& June } \\
\text { Average dissimilarity }=38.54\end{array}$} & \multicolumn{2}{|c|}{$\begin{array}{l}\text { Groups March \&June } \\
\text { Average dissimilarity }=30.59\end{array}$} & \multicolumn{2}{|c|}{$\begin{array}{l}\text { Groups April \& June } \\
\text { Average dissimilarity }=20.20\end{array}$} \\
\hline \multicolumn{2}{|c|}{$\begin{array}{l}\text { Groups May \&June } \\
\text { Average dissimilarity }=11.91\end{array}$} & \multicolumn{2}{|c|}{$\begin{array}{l}\text { Groups January \& July } \\
\text { Average dissimilarity }=50.77\end{array}$} & \multicolumn{2}{|c|}{$\begin{array}{l}\text { Groups February \& July } \\
\text { Average dissimilarity }=44.97\end{array}$} \\
\hline \multicolumn{2}{|c|}{$\begin{array}{l}\text { Groups March \& July } \\
\text { Average dissimilarity }=39.87\end{array}$} & \multicolumn{2}{|c|}{$\begin{array}{l}\text { Group April \& July } \\
\text { Average dissimilarity }=30.88\end{array}$} & \multicolumn{2}{|c|}{$\begin{array}{l}\text { Groups May \& July } \\
\text { Average dissimilarity }=23.20\end{array}$} \\
\hline
\end{tabular}




\begin{tabular}{|c|c|c|}
\hline $\begin{array}{l}\text { Groups June \& July } \\
\text { Average dissimilarity }=19.10\end{array}$ & $\begin{array}{l}\text { Groups January \& August } \\
\text { Average dissimilarity }=56.22\end{array}$ & $\begin{array}{l}\text { Groups February \& August } \\
\text { Average dissimilarity }=46.72\end{array}$ \\
\hline $\begin{array}{l}\text { Groups March \& August } \\
\text { Average dissimilarity }=42.96\end{array}$ & $\begin{array}{l}\text { Groups April \& August } \\
\text { Average dissimilarity }=36.20\end{array}$ & $\begin{array}{l}\text { Groups May \& August } \\
\text { Average dissimilarity }=28.06\end{array}$ \\
\hline $\begin{array}{l}\text { Groups June \& August } \\
\text { Average dissimilarity }=24.33\end{array}$ & $\begin{array}{l}\text { Groups July \& August } \\
\text { Average dissimilarity }=10.07\end{array}$ & $\begin{array}{l}\text { Groups January \& September } \\
\text { Average dissimilarity }=47.02\end{array}$ \\
\hline $\begin{array}{l}\text { Groups February \& September } \\
\text { Average dissimilarity }=40.66\end{array}$ & $\begin{array}{l}\text { Groups March \& September } \\
\text { Average dissimilarity }=36.32\end{array}$ & $\begin{array}{l}\text { Groups April \& September } \\
\text { Average dissimilarity }=34.14\end{array}$ \\
\hline $\begin{array}{l}\text { Groups May \& September } \\
\text { Average dissimilarity }=25.71\end{array}$ & $\begin{array}{l}\text { Groups June \& September } \\
\text { Average dissimilarity }=20.59\end{array}$ & $\begin{array}{l}\text { Groups July \& September } \\
\text { Average dissimilarity }=15.29\end{array}$ \\
\hline $\begin{array}{l}\text { Groups August } \& \text { September } \\
\text { Average dissimilarity }=18.83\end{array}$ & $\begin{array}{l}\text { Groups January \& October } \\
\text { Average dissimilarity }=42.25\end{array}$ & $\begin{array}{l}\text { Groups March \& October } \\
\text { Average dissimilarity }=33.77\end{array}$ \\
\hline $\begin{array}{l}\text { Groups April \& October } \\
\text { Average dissimilarity }=34.68\end{array}$ & $\begin{array}{l}\text { Groups May \& October } \\
\text { Average dissimilarity }=27.19\end{array}$ & $\begin{array}{l}\text { Groups June \& October } \\
\text { Average dissimilarity }=21.65\end{array}$ \\
\hline $\begin{array}{l}\text { Groups July \& October } \\
\text { Average dissimilarity }=23.1\end{array}$ & $\begin{array}{l}\text { Groups August \& October } \\
\text { Average dissimilarity }=26.39\end{array}$ & $\begin{array}{l}\text { Groups September \& October } \\
\text { Average dissimilarity }=12.56\end{array}$ \\
\hline $\begin{array}{l}\text { Groups March \& November } \\
\text { Average dissimilarity }=36.58\end{array}$ & $\begin{array}{l}\text { Groups April \& November } \\
\text { Average dissimilarity }=40.82\end{array}$ & $\begin{array}{l}\text { Groups May \& November } \\
\text { Average dissimilarity }=31.66\end{array}$ \\
\hline $\begin{array}{l}\text { Groups June \& November } \\
\text { Average dissimilarity }=31.97\end{array}$ & $\begin{array}{l}\text { Groups July \& November } \\
\text { Average dissimilarity }=33.04\end{array}$ & $\begin{array}{l}\text { Groups August \& November } \\
\text { Average dissimilarity }=38.03\end{array}$ \\
\hline $\begin{array}{l}\text { Groups September \& November } \\
\text { Average dissimilarity }=25.09\end{array}$ & $\begin{array}{l}\text { Groups October \& November } \\
\text { Average dissimilarity }=19.68\end{array}$ & $\begin{array}{l}\text { Groups January \& December } \\
\text { Average dissimilarity }=46.35\end{array}$ \\
\hline $\begin{array}{l}\text { Groups February \& December } \\
\text { Average dissimilarity }=43.27\end{array}$ & $\begin{array}{l}\text { Groups March \& December } \\
\text { Average dissimilarity }=43.93\end{array}$ & $\begin{array}{l}\text { Groups April \& December } \\
\text { Average dissimilarity }=50.12\end{array}$ \\
\hline $\begin{array}{l}\text { Groups May \& December } \\
\text { Average dissimilarity }=42.30\end{array}$ & $\begin{array}{l}\text { Groups June \& December } \\
\text { Average dissimilarity }=44.83\end{array}$ & $\begin{array}{l}\text { Groups July \& December } \\
\text { Average dissimilarity }=45.62\end{array}$ \\
\hline $\begin{array}{l}\text { Groups August \& December } \\
\text { Average dissimilarity }=49.46\end{array}$ & $\begin{array}{l}\text { Groups September \& December } \\
\text { Average dissimilarity }=40.75\end{array}$ & $\begin{array}{l}\text { Groups October \& December } \\
\text { Average dissimilarity }=38.97\end{array}$ \\
\hline \multicolumn{2}{|c|}{$\begin{array}{l}\text { Groups November \& December } \\
\text { Average dissimilarity }=22.59\end{array}$} & \\
\hline
\end{tabular}

\section{Fish diversity indices}

The mean Shannon-Wiener diversity index $\left(H^{\prime}\right)^{22}$ value was $2.28 \pm 0.294$ which ranged from 2.22 (January) to 3.29 (August) (Figure 3). The Margalef species richness $(d)^{23}$ ranged from 3.01 (January) to 7.63 (August) with a mean value of $5.18 \pm 1.23$ (Figure 3). The highest Pielou's ${ }^{24}$ evenness index $\left(J^{\prime}\right) 0.98$ was recorded in May and June; lowest 0.94 was in August and December with a mean value of $0.96 \pm 0.013$ (Figure 3). The peak Simpson Dominance index $(c)^{25}$ value 0.93 was observed in January and 0.97 in May to September with a mean value of $0.96 \pm 0.012$ (Figure 3). 


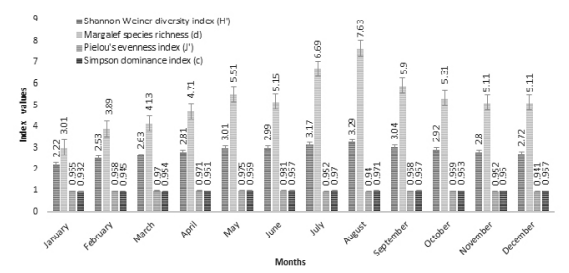

Figure 3: Different diversity index values in different months

Fish species assemblage

- Similarity Percentage (SIMPER) between months

SIMPER analysis divulged the average percent dissimilarity of species between different months. In the present study, highest dissimilarity (56.22\%) was observed in between January-August group and lowest $(10.07 \%)$ in the July-August group (Table 2). The highest contributing species in the January -August group were Mystus tengra (7.35\%), Chanda nama (6.73\%), Mystus tengara (6.52\%), Pethia ticto (5.30\%), Osteobrama cotio (4.96\%), Macrognathus aculeatus (4.54\%), Eutropiichthys vacha (4.54\%), Labeo calbasu (4.30\%), Ailia coila (4.30\%), Mystus bleekeri (4.08\%), Mastacembelus armatus (4.07\%), Puntius sophore (3.73\%), Dichotomyctere fluviatilis (3.70\%), Heteropneustes fossilis (3.33\%), Salmostoma acinaces (3.33\%), Amblypharyngodon mola (3.11\%), Channa gachua (2.86\%), Gudusia chapra (2.27\%), Corica soborna (1.87\%), Tenualosa ilisha (1.43\%) and Esomus danricus (1.43\%). On the other hand the contributing species in the July-August group were Heteropneustes fossilis (13.03\%), Mystus tengara (10.41\%), Mastacembelus armatus (7.05\%), Nandus nandus (5.61\%), Otolithoides pama (5.61\%), Plotosus canius (5.61\%), Conta conta (5.61\%), Ailia coila (5.61\%), Salmostoma acinaces (4.14\%), Puntius sophore (3.82\%), Rohtee cotio (3.66\%), Awaous grammepomus (3.28\%), Labeo bata (3.28\%), Dichotomyctere fluviatilis (3.28\%), Gudusia chapra (2.33\%), Channa gachua (2.33\%), Pethia ticto (2.12\%), Amblypharyngodon mola $(2.03 \%)$ and Eutropiichthys vacha (2.03\%).

\section{- Cluster analysis and Non-metric Multidimensional Scaling (nMDS)}

Four marked separation was observed in the abundance of fish in different months (figure 4). At the similarity of $72.9 \%$ fish species, four groups were attained in which January, February, March represents one cluster; April, May, June a second cluster and July, August, September, October a separate cluster. In November and December represents a single cluster during the research period.

(nMDS) Non-metric_Multidimensional scaling was employed to hit off abundance similarities among fish. Nonmetric_Multidimensional scaling shows $50 \%$ similarity for all months. On the other hand, three separate clustering showed in $70 \%$ similarity. However, at $80 \%$ similarity four separate clusters were observed in the fish abundance in the Ratargul swamp forest area (figure 5). 


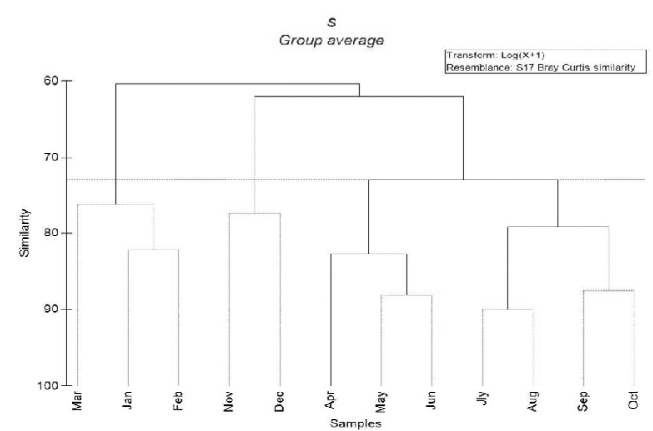

Figure 4: Cluster analysis based on Bray-Curtis similarity matrix of different months.

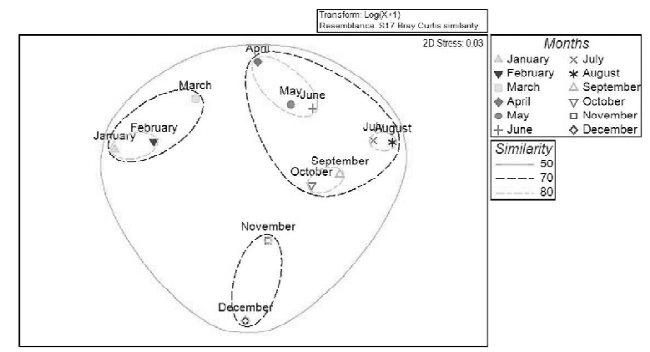

Figure 5: 2-dimensional ordination in nMDS showing similarity of different months

Swamp forest has a great ecological significance due to their unique characteristics in maintaining wetland biodiversity. Nishat ${ }^{30}$ mentioned Ratargul swamp forest as 'Bio-ecological zones of Bangladesh'. Ratargul swamp forest, Sylhet, was reported with 73 species plants, 20 species amphibians, 175 species of birds and 26 species of mammals by Choudhury ${ }^{31}$. After 12 years, Islam ${ }^{16}$ studied ichthyofaunal pattern in Ratargul swamp forest, they found only 12 numbers of fishes and the study period was only for one week. In our study, a total of 37 species of fishes were found from one-year time duration.

In the study time, order Cypriniformes dominated contributing $27.03 \%$ of the total fish assemblages (Figure 2) similar to the results from Hakaluki Haor, Sylhet ${ }^{9}$, in Upper Halda River, Chittagong ${ }^{32}$ and in River choto Jamuna of Naogaon ${ }^{7}$. In the present study, 14 threatened species (37.84\%) including endangered species five, Vulnerable seven species and Critically Endangered two fish species were found. Freshwater fish diversity in Bangladesh rich with 253 species, among these 64 are categorized as threatened (IUCN, 2015) ${ }^{33}$. The two Critically Endangered species of the swamp were Bagarius bagarius and Eutropiichthys vacha from the order Siluriformes.

Iqbal $^{9}$ recorded mean values of different indices: Shannon-Wiener diversity $\left(H^{\prime}\right)^{22}$, richness $(d)$, Evenness (e) and Dominance indices were as 2.53, 5.94, 0.66 and 0.081, respectively in Hakaluki Haor, Sylhet - while mean values were found 2.28 , 5.18, 0.96 and 0.96, respectively in Ratargul swamp forest. Shannon-Wiener diversity $\left(H^{\prime}\right)^{22}$, richness $(d)$ values of Ratargul swamp were lower than Hakaluki Haor, the reasons might be few numbers of fish species and less water spread area of swamp. Biligrami $^{34}$ recommended that ShannonWiener diversity index ${ }^{22}$ ranged from 3.04.50 , which represents good condition for fish diversity of particular waterbody. In present study, Shannon-Wiener diversity ${ }^{22}$ mean value was 2.28 , indicates the muddy condition of water body.

Magurran $^{35}$ stated that a biodiversity index is a single number which used to 
identifying the diversity of a specimen or species. Again, the two components, those are: the number of species or richness and the distribution of specimen among species mainly involve in the concept of species diversity ${ }^{36,37}$.

Shannon Wiener diversity index represents the proportion and richness of each species. Evenness and Dominance indices consider the contribution of common species and the relative number of individuals in the sample respectively.

Shannon-Wiener diversity index $\left(H^{\prime}\right)^{22}$ showed that fish assemblage was moderately diverse in Ratargul Swamp Forest. ShannonWiener diversity index $\left(H^{\prime}\right)^{22}$ and Margalef species richness $(d)^{23}$ index were observed high in August (3.29 and 7.63 respectively) while low in January month (2.22 and 3.01 respectively). In August, the Ratargul swamp area was inundated by floodwater and fish species were migrated to the swamp forest from Guine river. Again, in this month the native fishermen found more fish in their catch, so diversity showed peak this month. According to Pielou's evenness index ${ }^{24}$, fish species were more evenly distributed in May and June (0.98) compared to other months. Simpson dominance index was estimating the highest in June (0.97) which indicates species dominancy was peak in that month compared to other months.

In terms of the cluster analysis, two clusters were observed and in one cluster had majority in the Ratargul swamp forest. Two major clusters were also found in Meghna river estuary, Bangladesh by
Hossain $^{38}$. However, in Ratargul swamp, group A comprises the fish species of January, February and March month. Group B contains April, May, June, July, August, September, October, November and December and showed $72.9 \%$ similarity with the group A. In general, samples from first cluster (January, February and March) maintained a similarity which is also same for last two months (November and December). The Non-Metric Multidimensional Scaling (nMDS) analysis remarks four separate clusters at the $80 \%$ similarity in the Ratargul swamp forest; indicates the pattern of species abundance. The present study found maximum contributing species for months are similar but their number of percentages varies from one another.

According to Galib ${ }^{7}$, the River Choto Jamuna of Naogaon district was found two exotic fishes. Again, the Hakaluki Haor of Sylhet was recorded fish species including three exotic species such as Hypophthalmichthys molitrix (Silver carp), Oreochromis niloticus (Nile tilapia) and $O$. mossambicus (Mozambic tilapia) ${ }^{9}$. Konoskhaihaor, Northeast Bangladesh, was also found to have two exotic fish species ${ }^{10}$. Parvez $^{39}$ found 10 exotic species, namely Cyprinus carpio, Hypophthalmichthys nobilis, Barbonymus gonionotus, Ctenopharyngodon idella, Hypophthalmichthys molitrix, Mylopharyngodon piceus, Hypostomus plecostomus, Clarius gariepinus, Oreochromis mossambicus, and 
Pangasianodon hypopthalmus from three rivers Dhepa, Punarbhaba, and Atrai from northwestern part of Bangladesh. Exotic species are alarming if they are found in natural waterbodies; they compete with existing ichthyofaunal diversity and destroy the harmony of biodiversity. In this study, no exotic fish was found; it bears a mark of appreciation of the natural fish diversity of Ratargul. The reasons might be there were no hatcheries and aquaculture practices in surrounding areas.

\section{CONSERVATION AND RECOMMENDATIONS}

The "wise use", or "sustainable use" (Article 3 from the policies of National Wetland Policies' in the Ramsar Convention Secretariat, 2010) $)^{40}$ of this swamp forest, should be followed for the conservation of Ratargul wetland in Bangladesh. The importance of improvement and protection of the environment and biodiversity is crucial for all nations, so the Government of Bangladesh (GoB) inserted the section in the constitution as "the state shall endeavor to protect and improve the environments preserve and safeguard the natural resources, biodiversity, wetlands, forests and wildlife for the present and future citizens"41 (Bang. const. art. XVIII A, 2011). These lead us to recommend several conservation strategies to ensure the existing ichthyofauna of the Ratargul freshwater swamp forest. It has distinct flow regimes, so the average freshwater management plan will not be applicable here, the following points are needed to add in conservation strategies:

Freshwater swamp forest is recognized as valuable breeding ground and nursery regions for spawning and juvenile fishes by Thayer $^{42}$. So, fishing should be banned in the breeding season (July-August). Moreover, fish diversity showed the highest in these months. Monofilament Synthetic Nylon Fibre Nets or Current jals should banned, because juveniles stocks of different fishes are destroyed by it.

Tourist flow to the Ratargul swamp forest should be regulated and managed. Poor families dependent on fishing should be provided with alternative livelihood programs in fish breeding seasons. Use of insecticides and pesticides in the cropping system of the surrounding forest should be a minimum in doses to protect the adverse effects on fish species. To conserve available fish species, wise use of this wetland and the establishment of fish sanctuary is recommended.

\section{CONCLUSION}

Ratargul swamp forest is important ecosystem in the aspect of fish resources, it will act as a fish harbor especially for breeding, feeding and nursing ground for fishes. A sum of 37 species including 14 threatened species and no exotic fish were found in the study. Again, the biodiversity indices (Shannon-Wiener diversity index ${ }^{22}$ $\left(H^{\prime}\right)$, the Margalef species richness ${ }^{23}(d)$, the Pielou's evenness index ${ }^{24}\left(J^{\prime}\right)$ and the Simpson dominance index ${ }^{25}$ (c) were 
$2.28 \pm 0.294,5.18 \pm 1.23,0.96 \pm 0.013$ and $0.96 \pm 0.012$ ) will provide key information for the conservation strategies of the ichthyofaunal diversity, Ratargul swamp forest.

\section{ACKNOWLEDGEMENTS}

This study was undertaken through the project "Study of ichthyofaunal diversity and conservation strategy of the freshwater swamp Ratargul, Sylhet, Bangladesh" funded by Ministry of Science and Technology (MOST), Government of the People's Republic of Bangladesh under Operational Fund Research and Development (R \& D), 2014-2015.

\section{REFERENCES}

1. Hussain, M.G., \& M.A. Mazid, 2001. Genetic improvement and conservation of carp species in Bangladesh, Bangladesh Fisheries Research Institute.

2. Shamsuzzaman, M.M., M.M. Islam, N.J. Tania, M.A. Al-Mamun, P.P. Barman \& X. Xu, 2017. Fisheries resources of Bangladesh: Present status and future direction, Aquaculture and Fisheries, 2(4):145-156.

3. Nabahungu, N.L. \& S.M. Visser, 2011. Contribution of wetland agriculture to farmers' livelihood in Rwanda, Ecol Econ, 71:4-12.

4. Rashid, A.Z. \& M.M., 2014. A precious gift from nature. Dhaka tribune. Retrieved from www.dhakatribune.com/oped/2014/mar/22/ precious-gift-nature.

5. Jennings, S.F., J.L. Mélin, R.M. Blanchard, N.K. Forster, Dulvy \& R.W. Wilson, 2008. Globalscale predictions of community and ecosystem properties from simple ecological theory, Proc R Soc Lond, 275(1641):13751383.

6. Sarkar, U.K., A.K. Pathak, R.K. Sinha, K.
Sivakumar, A.K. Pandian, A. Pandey, V.K. Dubey \& W.S. Lakra, 2012. Freshwater fish biodiversity in the River Ganga (India): changing pattern, threats and conservation perspectives, Rev Fish Biol Fish, 22(1): 251272.

7. Galib, S.M., S.M.A. Naser, A.B.M. Mohsin, N. Chaki, M.F.H. Fahad, 2013. Fish diversity of the River Choto Jamuna, Bangladesh : present status and conservation needs, Int $J$ Biodivers Conserv, 5(6):389-395.

8. Rahman, M.M., M.Y. Hossain, F. Ahamed, B.R.F. Subba, E.M. Abdallah \& J. Ohtomi J, 2012. Biodiversity in the Padma Distributary of the Ganges River, Northwestern Bangladesh: Recommendations for Conservation, J. Zool., 7(4):328-337.

9. Iqbal, M.M., S. Nasren, M.A.A. Mamun \& M.M. Hossain, 2015a. Fish assemblage including threatened species in Hakaluki haor, Sylhet, Bangladesh, J. Aquacult. Trop., 30 (3-4):233246.

10. Iqbal, M.M., M.H. Kanon, M.A. Hossain, A. Hossain, S. Nasren, M.J. Islam \& M.A. Rahman, 2015b. Diversity of indigenous fish species in Konoskhaihaor, Northeast Bangladesh, Punjab Univ J Zool, 30(2):7379.

11. Hossain, M.A., M. Akter \& M.M. Iqbal, 2017. Diversity of fish fauna in Kusiara River (Fenchungonj Upazilla), Northeast Bangladesh, J. Aquacult. Trop., 32(1-2):113.

12. Johnson, J.A., R. Parmar, K. Ramesh, S. Sen \& R.S. Murthy, 2012. Fish diversity and assemblage structure in Ken River of Panna landscape, central India, J. Threat Taxa, 3161-3172.

13. Ramanujam, M.E., K.R. Devi \& T.J. Indra, 2014. Ichthyofaunal diversity of the Adyar Wetland complex, Chennai, Tamil Nadu, southern India, J. Threat Taxa, 6(4):5613-5635.

14. Bayçelebi, E, D. Turan \& B. Japoshvili, 2015. Fish fauna of Çoruh River and two first record for Turkey. Turkish J. Fish. Aquat. Sci., (15):783-794. 
15. Das, R.K., 2018. Fish diversity and the conservation status of a wetland of Cooch Behar District, West Bengal, India, J. Threat Taxa, 10(3):11423-11431.

16. Islam, M.S., M.A. Islam, N.A. Sweety, M.A.R. Hossain \& M.H. Kabir, 2016. Assessment of aquatic faunal diversity in the Ratargul swamp forest at Sylhet in Bangladesh, $J$. Environ. Sci. Nat. Resour., 9(2):51-64.

17. Bhuiyan, A.L., 1964. Fishes of Dacca, Publication No. 13, Dacca, The Asiatic Society of Pakistan, pp. 148.

18. Rahman, A.K.A., 1989. Freshwater Fishes of Bangladesh, ZSB, Department of Zoology, University of Dhaka, Dhaka-1000, pp. 161233.

19. Rahman, A.K.A., 2005. Freshwater Fishes of Bangladesh, 2nd edition, ZSB, Department of Zoology, University of Dhaka, Dhaka1000, pp. 263.

20. Talwar, P.K. \& A.G. Jhingran, 1991. Inland fishes of India and adjacent countries, New Delhi, India, Oxford and IBH Publishing Company Pvt, Ltd press, Vol. 1 and 2, pp. 1158.

21. Nelson, J.S. (eds.), 2006. Fishes of the World, John Wiley \& Sons, Inc, pp. 601.

22. Shannon, C.E. \& W. Weiver, 1949. The Mathematical Theory of Communication, Urbana, IL: University of Illinois Press, Urbana, pp. 117.

23. Margalef, R., 1968. Perspectives in Ecological Theory, Chicago II, Chicago, University of Chicago Press, pp. 111.

24. Pielou, E.C., 1966. Species diversity and pattern diversity in the study of ecological succession, J. Theor. Biol., 3:131-144.

25. Simpson, E.H., 1949. Measurement of diversity, Nature, 163:(688).

26. Clarke, K.R., 1993. Non parametric multivariate analyses of changes in community structure, Aust. J. Ecol., 18(1):117-143.

27. Clarke, K.R. \& R.M. Warwick, 1994. Similaritybased testing for community pattern: the 2way layout with no replication, Mar. Biol., 118:167-176.
28. Bray, J.R. \& J.C. Curtis, 1957. An ordination of the upland forest communities of southern Wisconsin, Ecol Monogr, 27:325-349.

29. Wellman, B., 1998. Doing it ourselves, Required reading, The SPSS manual as Sociology's most influential books. In Clawson, Dan. Required Reading: Sociology's most influential books, Amherst: University of Massachusetts press, pp. 71-78.

30. Nishat, A., S.I. Huq, S.P. Barua, A.H.M.A. Reza \& A.M. Khan, 2002. Bio-ecological zones of Bangladesh. The World Conservation Union (IUCN), Dhaka, Bangladesh, pp. 141.

31. Choudhury, J.K., S.R. Biswas, M.S. Islam, O. Rahman \& S.N. Uddin, 2004. Biodiversity of Ratargul swamp forest, Sylhet, IUCN Bangladesh Country Office, Dhaka, Bangladesh, pp. 4-24.

32. Alam, M.S., M.S. Hossain, M.M. Monwar \& M.E. Hoque, 2013. Assessment of fish distribution and biodiversity status in Upper Halda River, Chittagong, Bangladesh, Biodivers Conserv, 5(6):349-357.

33. (IUCN) International Union for Conservation of Nature, Red List of Bangladesh, Freshwater Fishes, International Union for Conservation of Nature, Dhaka, Bangladesh, 2015. Vol. 5, pp. 360.

34. Bilgrami, K.S., 1988. Biological Monitoring of Rivers-Problems and Prospects, Proc Indian National Sci., 5(2-3):171-174.

35. Magurran, A.E., 1988. Ecological Diversity and its Measurement, Princeton University press, Princetron, pp. 281.

36. Williamson, M., 1973. Species Diversity in ecological communities M S Bartlett, Horns R W (eds.). The Mathematical Theory of the Dynamics of Biological Populations, pp. 325336, London, (1973) Academic press, pp. 567.

37. Ponce-Hernandez, R., P. Koohafkan \& J. Antoine, 2004. Assessing carbon stocks and modelling win-win scenarios of carbon sequestration through land-use changes, Rome: Food and Agriculture Organization of the United Nations, Vol. 1. 
38. Hossain, M.S., N.G. Das, S. Sarkar \& M.Z. Rahaman, 2012. Fish diversity and habitat relationship with environmental variables at Meghna river estuary, Bangladesh, Egypt $J$ Aquat Res, 38(3):213-226.

39. Parvez, I., M.A. Alam, M.M. Hassan, Y. Ara, I. Hoshan \& A.S.M. Kibria, 2019. A checklist of fish species from three rivers in northwestern Bangladesh based on a sevenyear survey, J. Threat Taxa, 11(6):1378613794.

40. Ramsar Convention Secretariat (eds.), 2010. National Wetland Policies: Developing and implementing National Wetland Policies.
Ramsar handbooks for the wise use of wetlands. Ramsar Convention Secretariat, Gland, Switzerland.

41. Bang. 2011. Const. Art. XVIII A.

42. Thayer, G.W., H.H. Staurt, W.J. Kenworthy, J.F. Ustach \& A.B. Hall, 1978. Habitat values of salt marshes, mangroves, and seagrasses for aquatic organisms, In: Greeson, P.E. Clark, (eds.). Wetland functions and values: the state of our understanding. American Water Resources Association. USA, Minneapolis press, pp. 247. 
